\title{
Dementia

\section{Validity and Utility of the Center for Epidemiological Studies Depression Scale for Detecting Depression in Family Caregivers of Persons with Dementia}

\author{
Jiangbo Ying ${ }^{a}$ Philip Yap ${ }^{b, c}$ Mihir Gandhi ${ }^{d, e}$ Tau Ming Liewa, \\ aDepartment of Geriatric Psychiatry, Institute of Mental Health, Singapore, Singapore; \\ ${ }^{\text {b}}$ Department of Geriatric Medicine, Khoo Teck Puat Hospital, Singapore, Singapore; \\ ${ }^{c}$ Geriatric Education and Research Institute, Singapore, Singapore; ${ }^{d}$ Biostatistics, Singapore

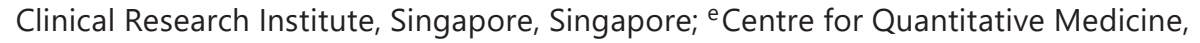 \\ Duke-NUS Medical School, Singapore, Singapore; fSaw Swee Hock School of Public Health, \\ National University of Singapore, Singapore, Singapore
}

\author{
Keywords \\ Dementia $\cdot$ Caregiver $\cdot$ Depression $\cdot$ Validity $\cdot$ Reliability $\cdot$ CES-D
}

\begin{abstract}
Background/Aims: The psychometric properties of Center for Epidemiological Studies Depression Scale (CES-D) have never been substantively investigated in caregivers of persons with dementia (PwD). We evaluated the validity and reliability of CES-D for detecting caregiver depression in dementia, and assessed whether CES-D could provide added utility beyond Zarit Burden Interview (ZBI). Method: Family caregivers of community-dwelling PwD $(n=394)$ completed self-administered questionnaires containing CES-D. Factorial validity was evaluated with confirmatory factor analysis; convergent and discriminant validity with Pearson's correlation coefficient; known-group validity by comparing across key variables; and internal consistency reliability with Cronbach's $\alpha$. Cohen's $\mathrm{k}$ was used to compare the agreement between those with depression (CES-D $\geq 16$ ) and those with high caregiver burden (ZBI >60). Results: CES-D demonstrated convergent, discriminant and known-group validity, consistent with a priori hypotheses. The original four-factor model of CES-D produced the best model-fit indices. Internal consistency reliability was good for the CES-D total scale $(\alpha=0.92)$, but lower for the Positive affect and Interpersonal problems subscales $(\alpha=0.70-0.74)$. Fortyfive percent of the caregivers had depression as identified by CES-D ( $95 \% \mathrm{Cl} 40-50 \%)$, but most of them were not identified by high ZBI scores $(\kappa=0.16)$. Conclusions: $C E S-D$ is a valid
\end{abstract}


Ying et al.: Caregiver Depression in Dementia

and reliable scale for detecting caregiver depression in dementia. It has added utility, beyond that of a caregiver burden scale, in identifying a subgroup of caregivers with depression but not burden. However, two subscales (Positive affect and Interpersonal problems) may require caution in interpretation among non-Caucasian caregivers.

() 2019 The Author(s)

Published by S. Karger AG, Basel

\section{Introduction}

Depression occurs in at least 1 in 3 caregivers of persons with dementia (PwD) (as demonstrated by a recent meta-analysis) [1], a prevalence which is comparatively higher than that in the general population or in the caregivers of persons with other physical or mental illnesses [1]. Depression can cause a variety of psychological and somatic problems and increase the risk of caregivers contemplating suicide [2]. It compromises caregivers' physical health [3], lowers caregivers' quality of life [4], and has been shown to cause the caregivers to place PwD in an institutional care facility more rapidly [5, 6]. Depression in caregivers can also impact the PwD adversely, as it has been associated with more rapid cognitive decline in PwD [7]. Considering the significance of this problem, it is pertinent to focus our efforts on case finding of caregiver depression with a validated scale.

While there are many scales to measure depression (such as 9-item Patient Health Questionnaire [PHQ-9] [8] and Geriatric Depression Scale [9]), the Center for Epidemiological Studies Depression Scale (CES-D) is one of the most widely-used scales in caregivers of PwD $[1,10,11]$ due to its simplicity of self-administration and the non-proprietary nature. In the literature, CES-D has demonstrated acceptable validity for both Western [12-17] and Asian populations [18-21], as well as for both general $[14,16]$ and clinical populations (such as in patients with cancer [15], diabetes [20], and schizophrenia [17]). The diagnostic accuracy of CES-D has also been confirmed in a recent meta-analysis [22], where it achieved an area under the curve of 0.87 on the summary receiver operating characteristic curve for the diagnosis of depression.

Notwithstanding the widespread use of CES-D, its psychometric properties have never been substantively investigated in caregivers of PwD. This is pertinent because the experience of depression in dementia caregiving can be qualitatively distinct from that of the other populations, often precipitated and perpetuated by the unique caregiving context related to the PwD. For example, in one study, caregivers of PwD reported that the sense of isolation as well as the physical and emotional demands of caring for PwD compounded their mental health issues [23]; while in another study, the caregivers reported the prominent theme of relational deprivations and losses, as well as the sentiments of powerlessness and helplessness [24]. These reports in the literature provided some examples on the unique experiences in caregiving which are less often endured by non-caregivers with depression.

Moreover, the literature has been unclear whether depression scales (such as CES-D) may have any added utility in dementia services. This is especially relevant considering that scales to capture caregiver stress/burden have already been routinely used in dementia services, and that depression and stress/burden are commonly conceptualized as falling within the same spectrum (with depression being the manifestation of high and significance stress/burden, as suggested by the diathesis-stress model [25]). As such, it remains uncertain whether the construct of depression would have been sufficiently measured by caregiver stress/burden scales, which essentially renders depression scales redundant in dementia caregiving. 
In this study, we sought to assess the validity (factorial validity, convergent and discriminant validity, and known-group validity) and reliability (internal consistency reliability) of CES-D for detecting depression in family caregivers of PwD. Essentially, our evaluations would include, among others, confirming the factor structure of CES-D in dementia caregivers, correlating CES-D with other scales of related constructs and elucidating the differences in CES-D scores across key demographic information (stage of dementia and behavioral problems of PwD). As a secondary aim, we also sought to assess whether CES-D provides any added utility beyond a commonly used caregiver burden scale (Zarit Burden Interview, ZBI) in identifying caregivers with significant duress.

\section{Methods}

\section{Participants and Procedures}

We recruited participants from the dementia clinics of two tertiary hospitals (Institute of Mental Health and Khoo Teck Puat Hospital). These two hospitals are the only two dementia services that serve the population in the North-East of Singapore, and hence provide a reasonably representative sampling of the population (given that majority of PwD in Singapore still received dementia care from tertiary hospitals) [26, 27]. At Institute of Mental Health, we also recruited participants from the inpatient wards of the geriatric psychiatry unit. These wards provide care for PwD with severe behavioral problems which are difficult to manage in the community settings. We used a consecutive sampling method and achieved a response rate of $87.8 \%$ in our recruitment.

The inclusion criteria comprised: (a) spouses or children of PwD; (b) caring for community-dwelling PwD (that is, the PwD were not dwelling in nursing homes); and (c) able to read in English or Chinese. The participants completed self-administered questionnaires which included a depression scale (CES-D) and a caregiver burden scale (ZBI). Participants from Khoo Teck Puat Hospital also completed an additional scale assessing caregiving gains (Gain in Alzheimer Care Instrument, GAIN).

This study received the ethical approval from the Domain Specific Review Board of National Healthcare Group, Singapore (Ref. No.: 2014/00127). It also received informed consent from all the participants.

\section{Measures}

CES-D is a 20 -item, self-administered scale which measures depressive symptoms in the previous 1 week [14]. The original author recommended a cut-off score of $\geq 16$ in CES-D to identify those with depression [14]. This cut-off score $(\geq 16)$ has also been validated in a recent meta-analysis, which demonstrated its sensitivity of 0.87 and specificity of 0.70 in detecting depression in the general population [22]. At the same time, an alternative cut-off score of $\geq 20$ was also proposed by the meta-analysis to balance between sensitivity and specificity ( 0.83 and 0.78 , respectively). CES-D has 4 subscales - Depressed affect, Somatic symptoms, Interpersonal problems, and Positive affect [14,28].

ZBI is a 22-item scale which measures the perceived caregiver stress/burden (caregiver burden has previously been defined as the physical, psychological, social, and financial problems of caring for an adult with a disabling condition) [29]. It has a total score ranging from 0 to 88, with scores above 60 indicating high caregiver burden [30]. ZBI has demonstrated good reliability and validity for assessing caregiver burden in various countries [31-33], including in Singapore [33, 34]. ZBI was shown to contain five subscales - Burden in the relationship, Emotional well-being, Social and family life, Finances, and Loss of control [35].

GAIN is a scale to measure caregiving gains in dementia. Higher scores denote higher gains. GAIN has previously been validated in Singapore [36, 37].

Demographic information related to caregivers and PwD was collected, based on self-reports by the caregivers or obtained from the medical records when the caregivers were uncertain. This information included age, gender, ethnic, marital status, employment status, highest education level, relationship with PwD, duration and frequency of caregiving, stage of dementia, and severe behavioral problems in PwD. The stage of dementia was obtained using a brief measure based on the three staging concept described in the revised third edition of Diagnostic and Statistical Manual of Mental Disorders (DSM-III-R) [38]. From the three options, participants chose the description that best described the PwD - still capable of independent living (mild stage), needs some assistance with daily living (moderate stage), or needs round-the-clock supervision (severe stage). This brief measure was previously shown to have reasonable agreement with the 
Ying et al.: Caregiver Depression in Dementia

Clinical Dementia Rating Scale ( $\kappa$ 0.56-0.6) [39-41]. The presence of severe behavioral problems was indirectly measured through the need for admission to the geriatric psychiatry ward. When PwD needed admission to the geriatric psychiatry ward, it indicated that PwD had behavioral problems that were too severe to be managed in the community setting.

\section{Statistical Analyses}

Overall, we conducted the following analyses: factorial validity, convergent and discriminant validity, known-group validity, and internal consistency reliability. The details of each analysis are described in the paragraphs below.

Factorial validity was assessed with confirmatory factor analysis [42, 43]. We compared the model fit between the original four-factor model $[14,28]$ and other alternative models in the literature [44-48]. We evaluated the goodness-of-fit of the models using root mean square error of approximation (RMSEA), standardized root mean square residual (SRMR), comparative fit index (CFI), and the Tucker-Lewis index (TLI). Good model fit was considered if the values of RMSEA and SRMR were below 0.08, and the values of CFI and TLI were above $0.9[42,43]$. We also used the Akaike information criterion in model comparison.

Convergent and discriminant validity was evaluated by examining the correlations among CES-D, ZBI, and their respective subscales, using the Pearson's correlation coefficient $(r)$. Correlation coefficients $>0.50$ were considered strong [49].

Known-group validity was assessed by comparing the mean scores of CES-D, using one-way analysis of variance. We pre-selected two variables (stage of dementia and behavioral problems of PwD) to contrast CES-D scores in the assessment of known-group validity, because more advanced dementia and the presence of behavioral problems in PwD have been reported as key risk factors for caregiver depression in a recent meta-analysis [10].

Internal consistency reliability of CES-D and its subscales was assessed using Cronbach's $\alpha$. The reliability indices of $\geq 0.70$ represent the minimally acceptable reliability to allow group comparisons in research settings [50,51], while values $\geq 0.90$ indicate suitability for individual-level measurements in clinical care [50-52]. For each item in the subscales of CES-D, we also tested the item-rest correlation and the Cronbach's $\alpha$ of the subscale if that item was removed from its subscale. The item-rest correlation of at least 0.40 [51] indicates the consistency of responses of each item with its counterparts within the same subscale. The Cronbach's $\alpha$ of a subscale should become lower if an item was deleted from the subscale, indicating the necessity of the item in maintaining internal consistency reliability.

As part of the secondary aim, we assessed whether high scores in a caregiver burden scale (ZBI) would have sufficed to identify those with significant burden and concomitant depression. Using Cohen's $\kappa$, we compared the agreement between those with ZBI scores of $>60$ (high caregiver burden) [30] and those with CES-D scores of $\geq 16$ (depression) [14, 22]. We also conducted sensitivity analysis to assess the changes in Cohen's $\kappa$ if the alternative cut-off score for CES-D $(\geq 20)[22]$ was used instead.

All analyses were performed using the STATA software version 13.

\section{Results}

We recruited 394 participants, with $54.6 \%$ of the participants from Institute of Mental Health while the rest from Khoo Teck Puat Hospital. The demographic information is shown in Table 1.

In confirmatory factor analysis, the original four-factor model of CES-D produced the best model fit indices (Table 2). This was followed closely by the three-factor model which had marginally poorer fit.

In the assessment of convergent and discriminant validity, the findings were consistent with what we had expected (Table 3) [36, 53-55]. CES-D correlated strongly with caregiver burden scale ZBI ( $r=0.71)$ and most of the subscales of ZBI $(r=0.60-0.70)$, but not with the Finances subscale of ZBI $(r=0.46)$ or the caregiving gains scale (GAIN) $(r=-0.16)$. Most of the subscales of CES-D correlated strongly with ZBI $(r=0.70-0.71)$, with the exception of the Interpersonal problems subscale and Positive affect subscale of CES-D, which correlated less strongly with ZBI ( $r=0.48$ and 0.29 , respectively). 
Dementia

Cognitive Disorders

Table 1. Demographic information on the caregivers and the persons with dementia ( $n=394)$

\begin{tabular}{l|l}
\hline Dement Geriatr Cogn Disord 2019;47:323-334 \\
\hline DOI: 10.1159/000500940 & $\begin{array}{l}\text { @ 2019 The Author(s). Published by S. Karger AG, Basel } \\
\text { www.karger.com/dem }\end{array}$ \\
\hline
\end{tabular}

Ying et al.: Caregiver Depression in Dementia
Variables related to caregivers

Age, years

Female gender

Ethnicity

Chinese

Malay

Indian

Others

Marital status

Married

Single

Widowed/divorced/separated

Employment status

Not working

Working part-time

Working full-time

Highest education

Primary or no formal education

Secondary

Tertiary

Relationship with PWD

Child

Spouse

Staying with PWD

Duration of caregiving in years

Frequency of caregiving

Daily, for at least $4 \mathrm{~h}$ a day

Daily, but less than $4 \mathrm{~h}$ a day

At least once a week

Less than once a week

Primary caregiver role

Variables related to PWD

Age, years

Female gender

Age at dementia diagnosis, years

Duration of dementia diagnosis, years

Stage of dementia ${ }^{a}$

Mild

Moderate

Severe

Severe behavioral problems ${ }^{b}$
$53.0 \pm 10.7$

236 (59.9)

$341(86.6)$

$25(6.3)$

$18(4.6)$

$10(2.5)$

$271(68.8)$

$94(23.9)$

$29(7.3)$

$123(31.2)$

$52(13.2)$

$219(55.6)$

$41(10.4)$

228 (57.9)

125 (31.7)

$340(86.3)$

54 (13.7)

$264(67.0)$

$6.8 \pm 6.7$

211 (53.6)

79 (20.0)

84 (21.3)

20 (5.1)

279 (70.8)

$79.5 \pm 8.2$

278 (70.6)

$75.6 \pm 8.5$

$4.5 \pm 3.5$

62 (15.7)

163 (41.4)

169 (42.9)

22 (5.6)

Data are presented as $n(\%)$ or mean \pm standard deviation. PWD, persons with dementia. ${ }^{a}$ The stage of dementia was obtained using a brief measure based on the three dementia severities described in the revised third edition of Diagnostic and Statistical Manual of Mental Disorders (DSM-III-R). From the three options, participants chose the description that best described the PWD - still capable of independent living (mild stage), needs some assistance with daily living (moderate stage), or needs round-the-clock supervision (severe stage). ${ }^{\mathrm{b}}$ The presence of severe behavioral problems was indirectly measured through the need for admission to a geriatric psychiatry ward, indicating a behavioral problem that was too severe to be managed in the community setting. 
Table 2. Fit indices of various models in confirmatory factor analysis $(n=394)$

\begin{tabular}{lllllll}
\hline Model & $\chi^{2}(\mathrm{df})$ & RMSEA $^{\mathrm{b}}$ & SRMR $^{\mathrm{b}}$ & CFI $^{\mathrm{b}}$ & TLI $^{\mathrm{b}}$ & AIC $^{\mathrm{c}}$ \\
\hline One-factor model $^{\mathrm{a}}$ & $895.00(170)$ & 0.104 & 0.071 & 0.828 & 0.808 & $16,503.58$ \\
Two-factor model $^{\mathrm{a}}$ & $605.02(169)$ & 0.081 & 0.051 & 0.896 & 0.884 & $16,215.59$ \\
Three-factor model $^{\mathrm{a}}$ & $557.54(167)$ & 0.077 & 0.049 & 0.907 & 0.894 & $16,172.12$ \\
Four-factor model (original) $^{\mathrm{a}}$ & $\mathbf{5 4 5 . 4 5 ( 1 6 4 )}$ & $\mathbf{0 . 0 7 7}$ & $\mathbf{0 . 0 4 9}$ & $\mathbf{0 . 9 0 9}$ & $\mathbf{0 . 8 9 5}$ & $\mathbf{1 6 , 1 6 6 . 0 2}$
\end{tabular}

The model which fulfilled the criteria of good fit is highlighted in bold. df, degree of freedom; RMSEA, root mean square error of approximation; SRMR, standardized root mean square residual; CFI, comparative fit index; TLI, Tucker-Lewis index; AIC, Akaike Information Criterion. ${ }^{a}$ The original four-factor model consisted of the subscales of Depressed affect, Somatic symptoms, Interpersonal problems, and Positive affect. The three-factor model comprises the Interpersonal problems subscale, Positive affect subscale, and a subscale that combines Depressed affect and Somatic symptoms. The two-factor model distinguishes the positive affect from the rest of the scale items. The one-factor model indicates CES-D as a unidimensional scale. ${ }^{\mathrm{b}} \mathrm{Criteria}$ for good model-fit: RMSEA $<0.08$, SRMR $<0.08$, CFI $>0.90$, TLI $>0.90$. ${ }^{c}$ Smaller AIC values indicate better model fit.

Table 3. Construct validity as shown by the correlation among various scales, using Pearson's correlation coefficient

\begin{tabular}{llllll}
\hline & \multicolumn{2}{l}{ CES-D and its subscales } & & \\
\cline { 2 - 6 } & $\begin{array}{l}\text { CES-D total } \\
\text { score }\end{array}$ & $\begin{array}{l}\text { Depressed } \\
\text { affect } \\
\text { subscale }\end{array}$ & $\begin{array}{l}\text { Somatic } \\
\text { symptoms } \\
\text { subscale }\end{array}$ & $\begin{array}{l}\text { Interpersonal } \\
\text { problems } \\
\text { subscale }\end{array}$ & $\begin{array}{l}\text { Positive } \\
\text { affect } \\
\text { subscale }\end{array}$ \\
\hline ZBI total scale & & 0.70 & 0.71 & $0.47^{\mathrm{d}}$ & $-0.29^{\mathrm{e}}$ \\
$\quad$ Burden in the relationship subscale & 0.60 & 0.59 & 0.63 & 0.39 & -0.20 \\
Emotional well-being subscale & 0.66 & 0.65 & 0.66 & 0.45 & -0.25 \\
Social and family life subscale & 0.65 & 0.62 & 0.64 & 0.44 & -0.30 \\
Loss of control subscale & 0.70 & 0.69 & 0.67 & 0.44 & -0.34 \\
Finances subscale & $0.46^{\mathrm{b}}$ & 0.47 & 0.45 & 0.29 & -0.18 \\
GAIN & $-0.16^{\mathrm{c}}$ & -0.11 & -0.04 & -0.07 & 0.30 \\
\hline
\end{tabular}

CES-D, Center for Epidemiological Studies Depression Scale; ZBI, Zarit Burden Interview; GAIN, Gain in Alzheimer Care Instrument. ${ }^{\text {a } C E S-D ~ c o r r e l a t e d ~ s t r o n g l y ~}(r>0.50)$ with a caregiver burden scale, ZBI. This is expected because the literature has reported the correlation between depression and burden in caregivers of those with other medical or psychiatric conditions $[54,55] .{ }^{b}$ CES-D correlated less strongly $(r \leq 0.50)$ with the Finances subscale of ZBI. The Finances subscale of ZBI, which comprises item 15 (do you feel that you don't have enough money to take care of your relative), is not expected to show concordance with the depressive symptoms measured by CES-D. ${ }^{c}$ CES-D correlated less strongly $(r \leq 0.50)$ with the GAIN scale. This is because GAIN, which measures positive outcomes in caregiving, is a different construct from the burden-depression spectrum and has only been shown to correlate weakly with ZBI [36]. ${ }^{\mathrm{d}}$ The Interpersonal problems subscale of CES-D correlated less strongly $(r \leq 0.50)$ with ZBI. The Interpersonal problems subscale of CES-D describes the feeling of critical reactions from

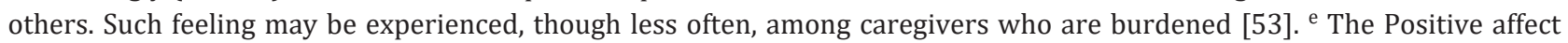
subscale of CES-D correlated less strongly $(r \leq 0.50)$ with ZBI because each of them measures a distinct construct.

In the assessment of known-group validity (Fig. 1), the findings were also consistent with what we had expected. The mean CES-D scores were significantly different among those caring for $\mathrm{PwD}$ at various stages of disease $(p=0.007)$. In pairwise comparisons, the mean CES-D scores were significantly higher among those caring for PwD at severe stage compared to the mild stage ( $p=0.035$ after Bonferroni adjustment) or the moderate stage ( $p=0.023$ after Bonferroni adjustment). However, the mean scores were not significantly different 


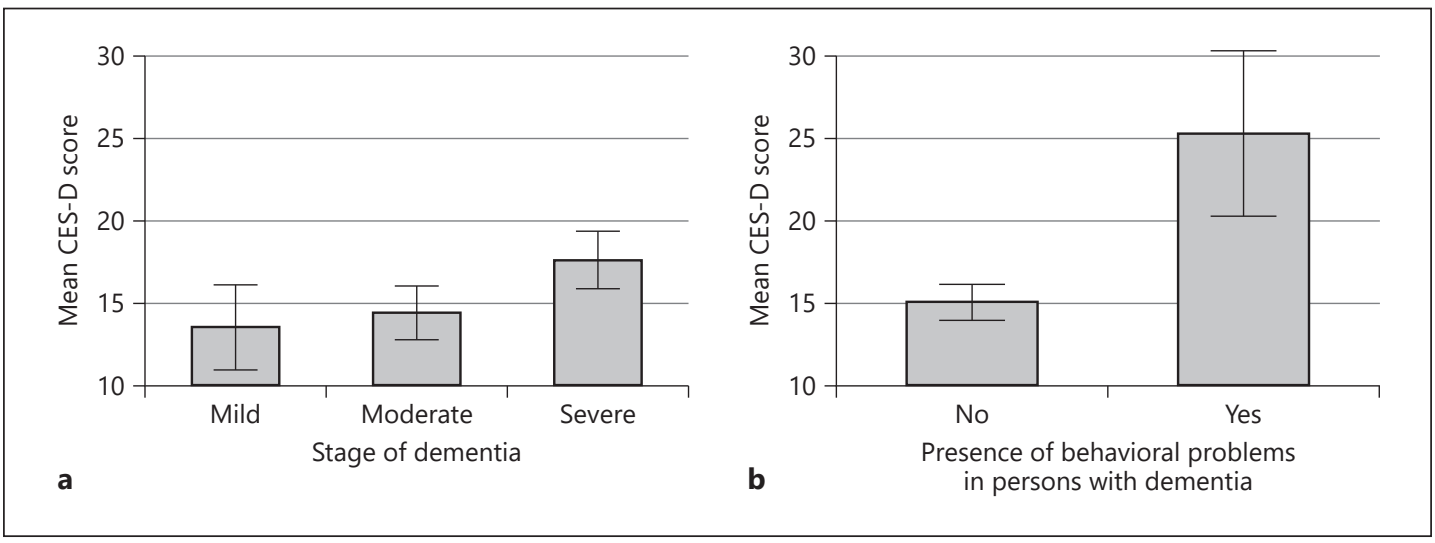

Fig. 1. Comparison of the mean scores of Center for Epidemiological Studies Depression Scale (CES-D) between groups which are known to affect the depressive symptoms in caregivers of persons with dementia in the assessment of known-group validity. a Mean scores of CES-D across the stages of dementia. $\mathbf{b}$ Mean scores of CES-D in the presence and absence of behavioral problems. Whiskers indicate the $95 \%$ confidence interval of the scores.

between the mild and moderate stage ( $p=1.000$ after Bonferroni adjustment). Similarly, caring for PwD with behavioral problems resulted in higher CES-D scores $(p<0.001)$.

Generally, CES-D and its subscales demonstrated good reliability. However, two of the subscales showed relatively lower reliability (Cronbach's $\alpha$ of 0.69 for the Interpersonal problems subscale; and 0.74 for the Positive affect subscale). Table 4 provides details on the internal consistency reliability of the individual items within each subscale. Most of the Cronbach's $\alpha$ were lowered if an item was deleted from a subscale, indicating the necessity of the item in maintaining internal consistency reliability.

The original cut-off score of CES-D $(\geq 16)$ identified $45 \%$ of the caregivers as having depression (95\% CI 40-50\%), while the alternative cut-off score $(\geq 20)$ identified a lower prevalence of 31\% (95\% CI 27-36\%). As shown in Figure 2, many caregivers with depression (CES-D $\geq 16$ ) did not have high ZBI scores $(>60)$. The agreement between high CES-D scores and high ZBI scores was modest $(\kappa=0.16)$. The agreement remained modest even when the alternative CES-D cut-off score of $\geq 20$ was used ( $\kappa=0.25$ ).

\section{Discussion}

Although CES-D has frequently been used to detect depression in caregivers of PwD, this is the first study, to our knowledge, that substantively explores the psychometric properties (factorial validity, convergent and discriminant validity, known-group validity, and internal consistency reliability) of CES-D in family caregivers of PwD. Similar to studies in non-caregivers $[19,20]$, the results give assurance to the validity and reliability of CES-D for detecting depression in caregivers of PwD. Our findings also support the original four-factor structure of CES-D as has been reported by previous studies involving caregivers of PwD [56-60]. Our reported prevalence of depression, in $31-45 \%$ of caregivers, was comparable with findings from the extant literature [1], which further strengthens our hypothesis that CES-D indeed measures the construct of depression in caregivers of PwD.

CES-D and all its subscales had reliability indices of near to or above 0.70 , indicating that they can be reliably used for group comparisons in research settings [50,51]. However, two subscales (Positive affect and Interpersonal problems) had relatively lower reliability (Cron- 
Table 4. Internal consistency reliability of the subscales of CES-D

\begin{tabular}{|c|c|c|}
\hline CES-D items within their original factors & $\begin{array}{l}\text { Item-rest } \\
\text { correlation }^{\mathrm{a}}\end{array}$ & $\begin{array}{l}\text { Cronbach's } \alpha \\
\text { if item deleted }^{b}\end{array}$ \\
\hline \multicolumn{3}{|l|}{ Subscale 1: Depressed affect (Cronbach's $\alpha=0.91$ ) } \\
\hline $\begin{array}{l}\text { 3. I felt that I could not shake off the blues even with the help } \\
\text { from my family or friends }\end{array}$ & 0.69 & 0.90 \\
\hline 6. I felt depressed & 0.82 & 0.88 \\
\hline 9. I thought my life had been a failure & 0.65 & 0.90 \\
\hline 10. I felt fearful & 0.79 & 0.89 \\
\hline 14. I felt lonely & 0.66 & 0.90 \\
\hline 17. I had crying spells & 0.67 & 0.90 \\
\hline 18. I felt sad & 0.77 & 0.89 \\
\hline \multicolumn{3}{|l|}{ Subscale 2: Somatic symptoms (Cronbach's $\alpha=0.85$ ) } \\
\hline 1. I was bothered by things that usually don't bother me & 0.66 & 0.82 \\
\hline 2. I did not feel like eating; my appetite was poor & 0.61 & 0.83 \\
\hline 5. I had trouble keeping my mind on what I was doing & 0.71 & 0.82 \\
\hline 7. I felt that everything I did was an effort & 0.57 & 0.84 \\
\hline 11. My sleep was restless & 0.62 & 0.83 \\
\hline 13. I talked less than usual & 0.47 & 0.85 \\
\hline 20. I could not get going & 0.67 & 0.82 \\
\hline \multicolumn{3}{|l|}{ Subscale 3: Positive affect (Cronbach's $\alpha=0.74$ ) } \\
\hline 4. I felt I was just as good as other people & 0.40 & 0.76 \\
\hline 8. I felt hopeful about the future & 0.52 & 0.69 \\
\hline 12. I was happy & 0.61 & 0.64 \\
\hline 16. I enjoyed life & 0.63 & 0.63 \\
\hline \multicolumn{3}{|l|}{ Subscale 4: Interpersonal problems (Cronbach's $\alpha=0.69$ ) } \\
\hline 15. People were unfriendly & 0.53 & NA \\
\hline 19. I felt that people dislike me & 0.53 & NA \\
\hline
\end{tabular}

CES-D, Center for Epidemiological Studies Depression Scale; NA, not applicable. ${ }^{a}$ Correlation between the item and the summated score for all other items from the same factor. ${ }^{b}$ Cronbach's $\alpha$ of the respective subscale if the item was deleted from the subscale.

bach's $\alpha$ 0.69-0.74) compared to the rest (Cronbach's $\alpha$ 0.85-0.92). With reliability indices which were far below the value of 0.90 , these two subscales may be less suitable for individual-level measurements in clinical care [50-52]. The more modest reliability results of these two subscales could possibly be due to the effects of culture, and suggest a need for more caution in interpreting these two subscales especially in non-Caucasian caregivers. The Positive affect subscale asks caregivers whether they feel good, hopeful, happy, and enjoy life. The items in this subscale might not be endorsed consistently in the oriental culture which emphasizes modesty and emotional restraint $[20,61,62]$. Such issue of internal consistency in the Positive affect subscale has been reported in a few studies involving Asian populations [20, 61, 62]. For instance, one study assessed CES-D among Korean immigrants in America and found that immigrants with lower adoption of the American culture reported less positive affect, with a noticeable dose-response relationship [61]. The influence of culture could also be seen with the Interpersonal problems subscale. The Interpersonal problems subscale focuses on the emotion of negative reactions from others. It is possible that the expression of such feelings may not be consistently endorsed in some populations, such as Singapore, where social harmony and respect for differences in personal beliefs are highly valued [63]. 
Fig. 2. Scatterplot between a commonly used caregiver burden scale (Zarit Burden Interview, ZBI) and Center for Epidemiological Studies Depression Scale (CES-D) $(n=394)$. ZBI scores above 40 are considered moderate to high caregiver burden (vertical line), while CES-D scores above 16 are considered significant depression (horizontal line).

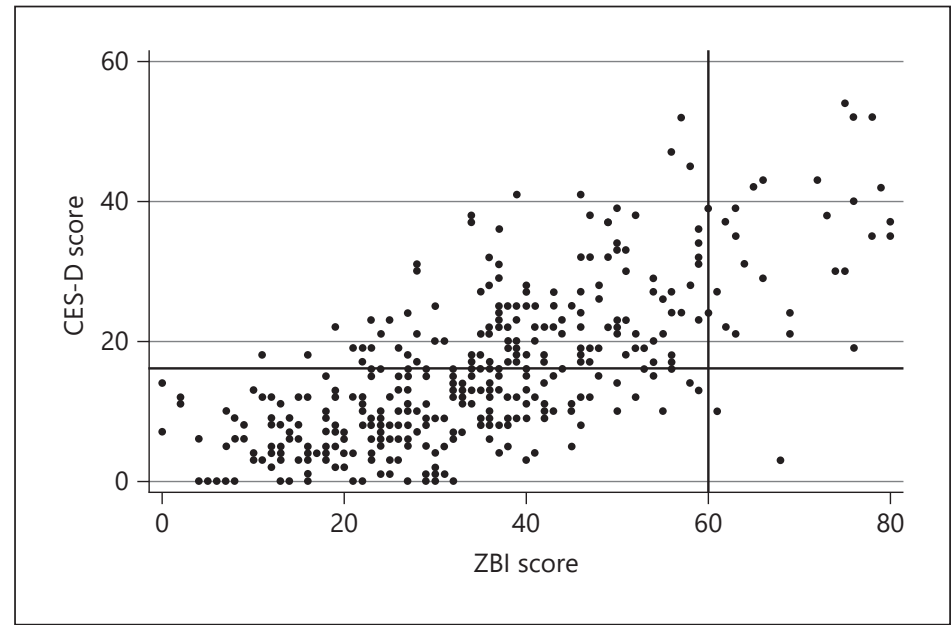

Conventionally, caregiver stress/burden and depression are perceived to mediate each other and fall within the same entity of duress as a result of caregiving [64]. As such, one would expect good agreement between those with high stress/burden and those with depression, and that a caregiver burden scale should suffice to identify those with depression. Unexpectedly, our findings suggest otherwise. While it is true that most of the caregivers with high burden also had depression (Fig. 2), the converse was not necessarily true. In this study, many caregivers with depression did not exhibit high burden. It is possible that this group of caregivers may have been depressed due to reasons beyond their caregiving experience. Another plausible explanation may be that these caregivers with depression are liable to selfblaming rather than attribute their predicament to caring for the PwD, which is not always socially sanctioned. This observation can benefit from future research to explore the caregiving experience of caregivers with depression in the absence of high burden, to ascertain the impact of depression on caregiving. The findings also demonstrate the added utility of CES-D beyond that of a caregiver burden scale, and reinforce the need to include assessment for depression in routine practice. This is important because the interventions for caregiver burden and depression can differ. Caregiver burden can be ameliorated by interventions that focus on improving the caregiving experience, such as psychoeducation, caregiver training, and respite care [65], while caregiver depression may require more intensive interventions (such as cognitive behavioral therapy) to target the mood symptoms of the caregiver [66].

Some limitations of this study should be mentioned. First, the scales were self-administered in this study; hence, caregivers with lower literacy might have been under-represented. Second, we did not directly measure the degree of behavioral problems in PwD and had only used the indirect measure of the need for admission to a geriatric psychiatry ward to capture PwD with the more severe degree of behavioral problems not manageable in the community setting. Third, this was a cross-sectional study, and we did not have the opportunity to assess longitudinal properties such as test-retest reliability or responsiveness. We are currently planning a separate longitudinal study to address this gap.

In conclusion, CES-D can be used in clinical care, as a valid and reliable scale, to detect caregiver depression in dementia. It has also demonstrated its usefulness, beyond that of a caregiver burden scale, in identifying a subgroup of caregivers with depression. However, two subscales of CES-D (Positive affect and Interpersonal problems) show more modest reliability, possibly due to the influence of culture, and may require more caution in interpretation when used among non-Caucasian caregivers. 


\section{Acknowledgement}

The authors thank the participants and the staff at Institute of Mental Health and Khoo Teck Puat Hospital, for their support in this research.

\section{Statement of Ethics}

This study received the ethical approval from the Domain Specific Review Board of National Healthcare Group, Singapore.

\section{Disclosure Statement}

There is no conflict of interest to declare.

\section{Funding Sources}

This work was supported by the Singapore Ministry of Health's National Medical Research Council under the Centre Grant Program (Grant No.: NMRC/CG/004/2013). It also received pilot funding from the National University of Singapore. Separately, the last author (T.M.L.) was supported by a research fellowship from the Singapore Ministry of Health's National Medical Research Council (Grant No.: NMRC/ Fellowship/0030/2016 and NMRC/CSSSP/0014/2017). The funding sources had no involvement in any part of the project.

\section{Author Contributions}

T.M.L. designed the study, planned the statistical analyses, collected the data, performed statistical analyses, interpreted the results, and wrote the manuscript. J.Y. searched the literature, interpreted the results, and contributed to the manuscript writing and revision. P.Y. advised on the study design, contributed to data collection, interpreted the results, and revised the manuscript. M.G. performed statistical analyses, interpreted the results, and reviewed the manuscript. All authors approved the final version of the manuscript for submission.

\section{References}

1 Sallim AB, Sayampanathan AA, Cuttilan A, Ho R. Prevalence of mental health disorders among caregivers of patients with Alzheimer Disease. J Am Med Dir Assoc. 2015 Dec;16(12):1034-41.

2 O’Dwyer ST, Moyle W, Zimmer-Gembeck M, De Leo D. Suicidal ideation in family carers of people with dementia. Aging Ment Health. 2016;20(2):222-30.

3 Pinquart M, Sörensen S. Correlates of physical health of informal caregivers: a meta-analysis. J Gerontol B Psychol Sci Soc Sci. 2007 Mar;62(2):126-37.

4 Bruvik FK, Ulstein ID, Ranhoff $\mathrm{AH}$, Engedal K. The quality of life of people with dementia and their family carers. Dement Geriatr Cogn Disord. 2012;34(1):7-14.

5 Coehlo DP, Hooker K, Bowman S. Institutional placement of persons with dementia: what predicts occurrence and timing? J Fam Nurs. 2007 May;13(2):253-77.

6 Luppa M, Luck T, Brähler E, König HH, Riedel-Heller SG. Prediction of institutionalisation in dementia. A systematic review. Dement Geriatr Cogn Disord. 2008;26(1):65-78.

7 Norton MC, Clark C, Fauth EB, Piercy KW, Pfister R, Green RC, et al. Caregiver personality predicts rate of cognitive decline in a community sample of persons with Alzheimer's disease. The Cache County Dementia Progression Study. Int Psychogeriatr. 2013 Oct;25(10):1629-37.

8 Kroenke K, Spitzer RL, Williams JB. The PHQ-9: validity of a brief depression severity measure. J Gen Intern Med. 2001 Sep;16(9):606-13.

9 Havins WN, Massman PJ, Doody R. Factor structure of the Geriatric Depression Scale and relationships with cognition and function in Alzheimer's disease. Dement Geriatr Cogn Disord. 2012;34(5-6):360-72. 
Ying et al.: Caregiver Depression in Dementia

10 Schoenmakers B, Buntinx F, Delepeleire J. Factors determining the impact of care-giving on caregivers of elderly patients with dementia. A systematic literature review. Maturitas. 2010 Jun;66(2):191-200.

11 Ying J, Yap P, Gandhi M, Liew TM. Iterating a framework for the prevention of caregiver depression in dementia: a multi-method approach. Int Psychogeriatr. 2018 Aug;30(8):1119-1130.

12 González P, Nuñez A, Merz E, Brintz C, Weitzman O, Navas EL, et al. Measurement properties of the Center for Epidemiologic Studies Depression Scale (CES-D 10): findings from HCHS/SOL. Psychol Assess. 2017 Apr;29(4):372-81.

13 McCallum J, Mackinnon A, Simons L, Simons J. Measurement properties of the Center for Epidemiological Studies Depression Scale: an Australian community study of aged persons. J Gerontol B Psychol Sci Soc Sci. 1995 May;50(3):S182-9.

14 Radloff LS. The CES-D Scale - A self-report depression scale for research in the general population. Appl Psychol Meas. 1977;1(3):385-401.

15 Chhabria KS, Carnaby GD. Psychometric validation of the Center for Epidemiological Studies Depression Scale in Head and Neck Cancer patients. Oral Oncol. 2017 Dec;75:158-62.

16 Head J, Stansfeld SA, Ebmeier KP, Geddes JR, Allan CL, Lewis G, et al. Use of self-administered instruments to assess psychiatric disorders in older people: validity of the General Health Questionnaire, the Center for Epidemiologic Studies Depression Scale and the self-completion version of the revised Clinical Interview Schedule. Psychol Med. 2013 Dec;43(12):2649-56.

17 Herniman SE, Allott KA, Killackey E, Hester R, Cotton SM. The psychometric validity of the Center for Epidemiological Studies - Depression Scale (CES-D) in first episode schizophrenia spectrum. Psychiatry Res. 2017 Jun;252:16-22.

18 Cheung CK, Bagley C. Validating an American scale in Hong Kong: the Center for Epidemiological Studies Depression Scale (CES-D). J Psychol. 1998 Mar;132(2):169-86.

19 Chin WY, Choi EP, Chan KT, Wong CK. The Psychometric Properties of the Center for Epidemiologic Studies Depression Scale in Chinese Primary Care Patients: Factor Structure, Construct Validity, Reliability, Sensitivity and Responsiveness. PLoS One. 2015 Aug;10(8):e0135131.

20 Zhang Y, Ting RZ, Lam MH, Lam SP, Yeung RO, Nan H, et al. Measuring depression with CES-D in Chinese patients with type 2 diabetes: the validity and its comparison to PHQ-9. BMC Psychiatry. 2015 Aug;15(1):198.

21 Stahl D, Sum CF, Lum SS, Liow PH, Chan YH, Verma S, et al. Screening for depressive symptoms: validation of the center for epidemiologic studies depression scale (CES-D) in a multiethnic group of patients with diabetes in Singapore. Diabetes Care. 2008 Jun;31(6):1118-9.

22 Vilagut G, Forero CG, Barbaglia G, Alonso J. Screening for Depression in the General Population with the Center for Epidemiologic Studies Depression (CES-D): A Systematic Review with Meta-Analysis. PLoS One. 2016 May; 11(5):e0155431.

23 O'Dwyer S, Moyle W, van Wyk S. Suicidal ideation and resilience in family carers of people with dementia: a pilot qualitative study. Aging Ment Health. 2013;17(6):753-60.

24 Vasileiou K, Barnett J, Barreto M, Vines J, Atkinson M, Lawson S, et al. Experiences of loneliness associated with being an informal caregiver: A Qualitative Investigation. Front Psychol. 2017 Apr;8:585.

25 Patten SB. Major depression epidemiology from a diathesis-stress conceptualization. BMC Psychiatry. 2013 Jan;13(1):19.

26 Liew TM, Yeap BI, Koh GC, Gandhi M, Tan KS, Luo N, et al. Detecting predeath grief in family caregivers of persons with dementia: Validity and utility of the Marwit-Meuser Caregiver Grief Inventory in a multiethnic Asian population. Gerontologist. 2018 Mar;58(2):e150-9.

27 Liew TM, Yap P, Luo N, Hia SB, Koh GC, Tai BC. Detecting pre-death grief in family caregivers of persons with dementia: measurement equivalence of the Mandarin-Chinese version of Marwit-Meuser caregiver grief inventory. BMC Geriatr. 2018 May;18(1):114.

28 Shafer AB. Meta-analysis of the factor structures of four depression questionnaires: Beck, CES-D, Hamilton, and Zung. J Clin Psychol. 2006 Jan;62(1):123-46.

29 George LK, Gwyther LP. Caregiver well-being: a multidimensional examination of family caregivers of demented adults. Gerontologist. 1986 Jun;26(3):253-9.

30 Zarit SH, Zarit JM. Instructions for the Burden Interview. University Park (PA): Pennsylvania State University; 1987.

31 Braun M, Scholz U, Hornung R, Martin M. [Caregiver burden with dementia patients. A validation study of the German language version of the Zarit Burden Interview]. Z Gerontol Geriatr. 2010 Apr;43(2):111-9.

32 Chattat R, Cortesi V, Izzicupo F, Del Re ML, Sgarbi C, Fabbo A, et al. The Italian version of the Zarit Burden interview: a validation study. Int Psychogeriatr. 2011 Jun;23(5):797-805.

33 Seng BK, Luo N, Ng WY, Lim J, Chionh HL, Goh J, et al. Validity and reliability of the Zarit Burden Interview in assessing caregiving burden. Ann Acad Med Singapore. 2010 0ct;39(10):758-63.

$34 \mathrm{Yu}$ J, Yap P, Liew TM. The optimal short version of the Zarit Burden Interview for dementia caregivers: diag nostic utility and externally validated cutoffs. Aging Ment Health. 2019 Jun;23(6):706-710.

35 Rankin ED, Haut MW, Keefover RW, Franzen MD. The establishment of clinical cutoffs in measuring caregiver burden in dementia. Gerontologist. 1994 Dec;34(6):828-32.

36 Yap P, Luo N, Ng WY, Chionh HL, Lim J, Goh J. Gain in Alzheimer care INstrument-a new scale to measure caregiving gains in dementia. Am J Geriatr Psychiatry. 2010 Jan;18(1):68-76.

37 Liew TM, Luo N, Ng WY, Chionh HL, Goh J, Yap P. Predicting gains in dementia caregiving. Dement Geriatr Cogn Disord. 2010;29(2):115-22. 
38 American Psychiatric Association. Diagnostic and Statistical Manual of Mental Disorders: DSM-III-R. Washington: American Psychiatric Association; 1987.

39 Morris JC. The Clinical Dementia Rating (CDR): current version and scoring rules. Neurology. 1993 Nov; 43(11):2412-4.

40 Forsell Y, Fratiglioni L, Grut M, Viitanen M, Winblad B. Clinical staging of dementia in a population survey: comparison of DSM-III-R and the Washington University Clinical Dementia Rating Scale. Acta Psychiatr Scand. 1992 Jul;86(1):49-54.

41 Juva K, Sulkava R, Erkinjuntti T, Ylikoski R, Valvanne J, Tilvis R. Staging the severity of dementia: comparison of clinical (CDR, DSM-III-R), functional (ADL, IADL) and cognitive (MMSE) scales. Acta Neurol Scand. 1994 Oct; 90(4):293-8.

$42 \mathrm{Hu}$, Bentler PM. Cutoff criteria for fit indexes in covariance structure analysis: conventional criteria versus new alternatives. Struct Equ Modeling. 1999;6(1):1-55.

43 Marsh HW, Hau K, Grayson D. Goodness of fit in structural equation models. In McArdle JJ, editor. Contemporary Psychometrics: A Festschrift for Roderick P. McDonald. Mahwah: Lawrence; 2005. p. 275-340.

44 Guarnaccia PJ, Angel R, Worobey JL. The factor structure of the CES-D in the Hispanic Health and Nutrition Examination Survey: the influences of ethnicity, gender and language. Soc Sci Med. 1989;29(1):85-94.

45 Zhang J, Sun W, Kong Y, Wang C. Reliability and validity of the Center for Epidemiological Studies Depression Scale in 2 special adult samples from rural China. Compr Psychiatry. 2012 Nov;53(8):1243-51.

46 Lacasse JJ, Forgeard MJ, Jayawickreme N, Jayawickreme E. The factor structure of the CES-D in a sample of Rwandan genocide survivors. Soc Psychiatry Psychiatr Epidemiol. 2014 Mar;49(3):459-65.

47 Schroevers MJ, Sanderman R, van Sonderen E, Ranchor AV. The evaluation of the Center for Epidemiologic Studies Depression (CES-D) scale: depressed and Positive Affect in cancer patients and healthy reference subjects. Qual Life Res. 2000;9(9):1015-29.

48 Wood AM, Taylor PJ, Joseph S. Does the CES-D measure a continuum from depression to happiness? Comparing substantive and artifactual models. Psychiatry Res. 2010 May;177(1-2):120-3.

49 Juniper EF, Guyatt GH, Jaeschke R. How to develop and validate a new health-related quality of life instrument. In Spiker B, editor. Quality of Life and Pharmacoeconomics in Clinical Trials. Philadelphia: Lippincott-Raven; 1996.

50 Nunnally JC, Bernstein IH. Psychometric theory. New York: McGraw-Hill; 1994.

51 Fayers PM, Machin D. Quality of life: The assessment, analysis and interpretation of patient-reported outcomes. Chichester: Wiley; 2007.

52 Weiner EA, Stewart BJ. Assessing individuals: Psychological and educational tests and measurements. Boston: Little, Brown; 1984.

53 Zarit SH, Reever KE, Bach-Peterson J. Relatives of the impaired elderly: correlates of feelings of burden. Gerontologist. 1980 Dec;20(6):649-55.

54 Perlick DA, Berk L, Kaczynski R, Gonzalez J, Link B, Dixon L, et al. Caregiver burden as a predictor of depression among family and friends who provide care for persons with bipolar disorder. Bipolar Disord. 2016 Mar; 18(2):183-91.

55 Oh J, An JW, Oh KW, Oh SI, Kim JA, Kim SH, et al. [Depression and caregiving burden in families of patients with amyotrophic lateral sclerosis]. J Korean Acad Nurs. 2015 Apr;45(2):202-10.

56 O'Rourke N. Equivalence of French and English language versions of the Center for Epidemiologic StudiesDepression (CES-D) among caregivers of persons with dementia. Can J Aging. 2003;22(3):323-9.

57 O'Rourke N. Factor structure of the Center for Epidemiologic Studies-Depression Scale (CES-D) among older men and women who provide care to persons with dementia. Int J Test. 2005;5(3):265-77.

58 Losada A, de los Angeles Villareal M, Nuevo R, Márquez-González M, Salazar BC, Romero-Moreno R, et al. Cross-cultural confirmatory factor analysis of the CES-D in Spanish and Mexican dementia caregivers. Span J Psychol. 2012 Jul;15(2):783-92.

59 Flynn Longmire CV, Knight BG. Confirmatory factor analysis of the Center for Epidemiologic StudiesDepression Scale in black and white dementia caregivers. Aging Ment Health. 2010 Nov;14(8):962-70.

60 Roth DL, Ackerman ML, Okonkwo OC, Burgio LD. The four-factor model of depressive symptoms in dementia caregivers: a structural equation model of ethnic differences. Psychol Aging. 2008 Sep;23(3):567-76.

61 Kim E, Seo K, Cain KC. Bi-dimensional acculturation and cultural response set in CES-D among Korean immigrants. Issues Ment Health Nurs. 2010 Sep;31(9):576-83.

62 Li Z, Hicks MH. The CES-D in Chinese American women: construct validity, diagnostic validity for major depression, and cultural response bias. Psychiatry Res. 2010 Feb;175(3):227-32.

63 Tan KB. Harmony as ideology, culture, and control: alternative dispute resolution in Singapore. Austral J Asian Law. 2007;9(1):120-51.

64 Clyburn LD, Stones MJ, Hadjistavropoulos T, Tuokko H. Predicting caregiver burden and depression in Alzheimer's disease. J Gerontol B Psychol Sci Soc Sci. 2000 Jan;55(1):S2-13.

65 Torti FM Jr, Gwyther LP, Reed SD, Friedman JY, Schulman KA. A multinational review of recent trends and reports in dementia caregiver burden. Alzheimer Dis Assoc Disord. 2004 Apr-Jun;18(2):99-109.

66 Weinbrecht A, Rieckmann N, Renneberg B. Acceptance and efficacy of interventions for family caregivers of elderly persons with a mental disorder: a meta-analysis. Int Psychogeriatr. 2016 Oct;28(10):1615-29. 